\title{
Nanomechanical spectroscopy of ultrathin silicon nitride suspended membranes ${ }^{\star}$
}

\author{
Sanket S. Jugade, Anuj Aggarwal, and Akshay K. Naik* \\ Centre for Nano Science and Engineering (CeNSE), Indian Institute of Science (IISc) Bengaluru 560012, India
}

Received: 23 March 2021 / Accepted: 29 April 2021

\begin{abstract}
Mechanical properties of a nanomechanical resonator significantly impact the performance of a resonant Nano-electromechanical system (NEMS) device. We study the mechanical properties of suspended membranes fabricated out of low-pressure chemical vapor deposited silicon nitride thin films. We fabricated doubly-clamped membranes of silicon nitride with thickness less than $50 \mathrm{~nm}$ and length varying from 5 to $60 \mu \mathrm{m}$. The elastic modulus and stress in the suspended membranes were measured using Atomic Force Microscope (AFM)-based nanomechanical spectroscopy. The elastic moduli of the suspended membranes are significantly higher than those of corresponding on-substrate thin films. We observed a reduction in net stress after the fabrication of suspended membrane, which is explained by estimating the thermal stress and intrinsic stress. We also use a mathematical model to study the stress and thickness-dependent elastic modulus of the ultrathin membranes. Lastly, we study the capillary force-gradient between the $\mathrm{SiN}_{\mathrm{x}}$ suspended membrane-Si substrate that could collapse the suspended membrane.
\end{abstract}

\section{Introduction}

Silicon nitride nanomechanical resonators have potential applications as Nano-electromechanical systems (NEMS) sensors [1-4] due to their high-quality factor (in the order of few millions) and high sensitivity at room temperatures [5-8]. The fundamental natural frequency of a doublyclamped membrane resonator depends on its elastic modulus and stress, as given below [9].

$$
f_{0}=1.03 \sqrt{\frac{E t^{2}}{\rho L^{4}}+\frac{\sigma W t}{3.4 \mathrm{ml}}}
$$

where $E$ is the elastic modulus, $\sigma$ is the stress in the suspended membrane, $\rho$ is the density of the material, $m, L$, $w$, and $t$ are the effective mass, length, width, and thickness of the suspended membrane respectively. Verbridge et al. [10] have shown that the addition of tensile stress to the silicon nitride NEMS resonators leads to higher quality factors, leading to better sensitivity. Furthermore, if the elastic modulus and stress in the suspended membrane are very low, they are likely to collapse or undergo plastic deformation and fracture under a few nano newtons of force. For thin films grown on a substrate, the mechanical properties can vary significantly from that of the bulk material, depending on the growth conditions [11-13]. The Low-Pressure Chemical Vapor Deposition (LPCVD) grown silicon nitride thin films studied in this work have a

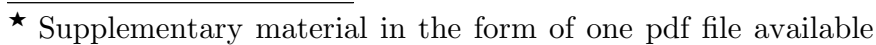
from the Journal web page at https://www.epjap.org/10.1051/ epjap/2021210068/olm

* e-mail: anaik@iisc.ac.in
}

thickness of less than $100 \mathrm{~nm}$ and are amorphous [14]. Residual stress in thin films depends on the growth conditions such as pressure, temperature, and gas flow ratio. The stress in an amorphous thin film has two components: intrinsic and thermal stress [15]. Intrinsic stress results from defects such as voids, porosities, impurities, and atomic diffusion. While thermal stress results from thermal expansion coefficient mismatch between the film and the substrate material [16]. High tensile stress in the deposited film leads to cracks in the film and subsequent failure of the fabricated suspended membrane. Hence, it becomes essential to measure the elastic modulus and stress in silicon nitride thin films on a substrate and suspended membranes fabricated out of them.

In this paper, first, we describe the fabrication of silicon nitride suspended membranes. Then we explain AFMbased elastic modulus and stress measurements in the $\mathrm{SiN}_{\mathrm{x}}$ suspended membranes. The stress in the suspended membranes is studied based on the relaxation of stress in the corresponding on-substrate thin films. While the variation of elastic modulus with the thickness of the suspended membrane is analyzed using a model based on surface-energy considerations. In the end, the capillary force-gradient between the suspended membrane and bottom substrate is modeled based on the experimental force-separation curves for the collapse of suspended membranes.

\section{Experimental procedures}

\subsection{Fabrication of suspended $\mathrm{SiN}_{\mathrm{x}}$ membranes}

We deposit LPCVD silicon nitride films of thickness 47, 51, and $88 \mathrm{~nm}$ on a $\mathrm{Si} / \mathrm{SiO}_{2}$ substrate. The process flow for 


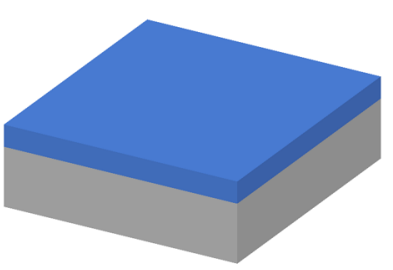

(i) Cleaning of $\mathrm{Si} / \mathrm{SiO}_{2}$ substrate

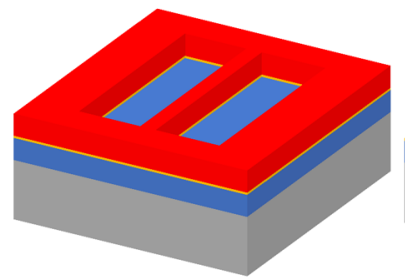

(iv) Reactive ion etching of $\operatorname{SiN}_{x}$

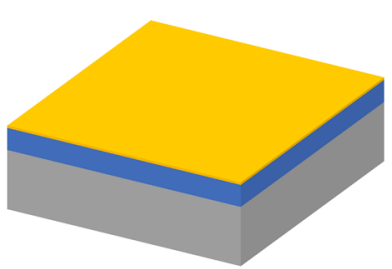

(ii) $\mathrm{SiN}_{\mathrm{x}}$ deposition

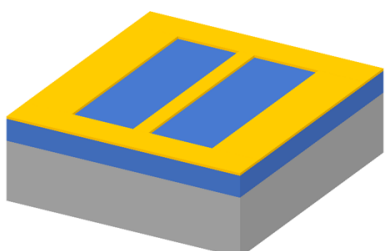

(v) Photoresist removal

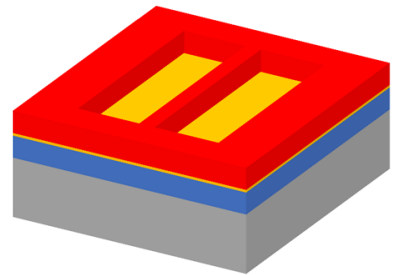

(iii) Electron beam lithography

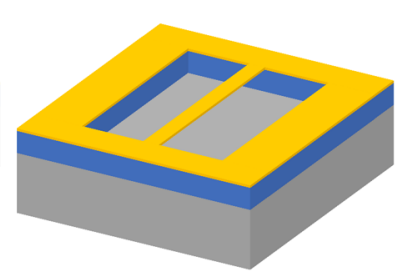

(vi) Wet etching of $\mathrm{SiO}_{2}$ and critical point drying

(a)

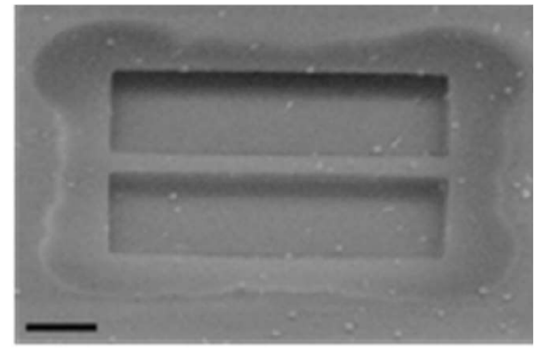

(b)

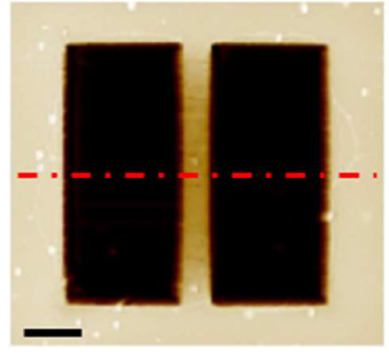

(c)

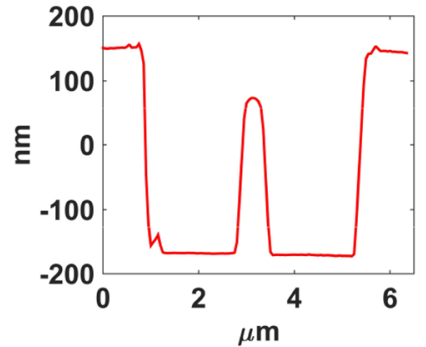

(d)

Fig. 1. (a) Process flow for the fabrication of a doubly-clamped silicon nitride membrane. (b and (c) Scanning Electron Micrograph and AFM image of a $5 \mu \mathrm{m}$ long and $500 \mathrm{~nm}$ wide suspended membrane, respectively. (d) Topography of the section shown in Figure 1c. Scale bar is $1 \mathrm{um}$ for Figures $1 \mathrm{~b}$ and $1 \mathrm{c}$.

fabrication of doubly-clamped $\mathrm{SiN}_{\mathrm{X}}$ membranes is illustrated in Figure 1a and briefly described in Supplementary Information. Figures $1 \mathrm{~b}$ and 1c show a Scanning Electron Microscope (SEM) and Atomic Force Microscope (AFM) micrographs of a suspended membrane, respectively. The suspended membrane shown in the figure is $5 \mu \mathrm{m}$ in length and $500 \mathrm{~nm}$ in width. To prevent the collapse of suspended membranes due to the charging effect, we did not perform SEM imaging of the actual suspended membranes of larger width used for the AFM measurements.

\subsection{Elastic modulus and stress measurement on suspended $\mathrm{SiN}_{\mathrm{x}}$ membranes}

We measured the elastic modulus and stress in the suspended membrane using the AFM-based central indentation method $[9,17,18]$. Central indentation experiments were performed with AFM using force-distance (F-d) spectroscopy [19-21]. The central indentation method is illustrated in Figure 2a. Figure 2c shows an AFM topography of a $5 \mu \mathrm{m}$ long, $1.3 \mu \mathrm{m}$ wide suspended membrane fabricated from a $51 \mathrm{~nm}$ thick silicon nitride film on a substrate. We obtain a Force vs. Deformation curve (Fig. 2d) after central indentation on this suspended membrane. We fit this curve using a force-deformation relation given in equation (2) for a doubly clamped membrane subjected to central loading [22] with $E$ and $\sigma$ as the fitting parameters.

$$
F=\left(16.23 E W\left(\frac{t}{L}\right)^{3}+4.93 \sigma \frac{W t}{L}\right) \delta+12.17 E \frac{W t}{L^{3}} \delta^{3}
$$

where $E$ is the elastic modulus of the suspended membrane, $\sigma$ is the stress in the suspended membrane, $\delta$ is the deformation in the suspended membrane, $L, W$ and $t$ are the length, width, and thickness of the suspended membrane. The coefficients of $\delta$ and $\delta^{3}$ terms in equation (2) include elastic modulus and stress. We performed the elastic modulus and stress measurements on three different membranes of $5 \mu \mathrm{m}$ length for each thickness of the suspended membranes.

\subsection{Elastic modulus and stress measurement on on-substrate $\mathrm{SiN}_{x}$ thin films}

We also measured the elastic modulus of the silicon nitride thin films on $\mathrm{Si} / \mathrm{SiO}_{2}$ substrate using the nanoindentation technique [23]. We performed the nanoindentation measurements at four different locations on each of the 

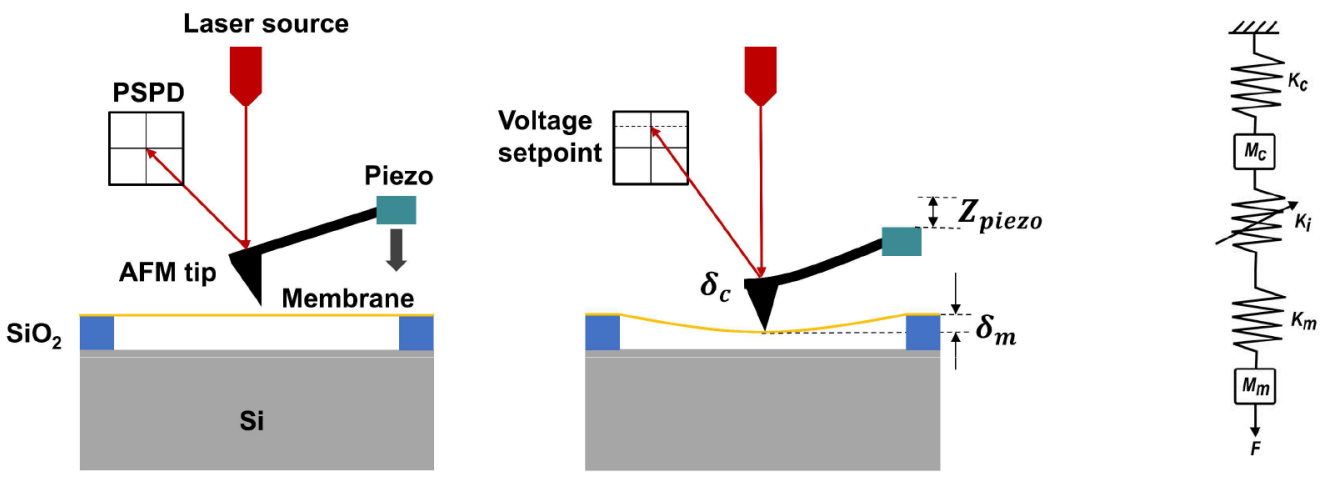

(a)

(b)

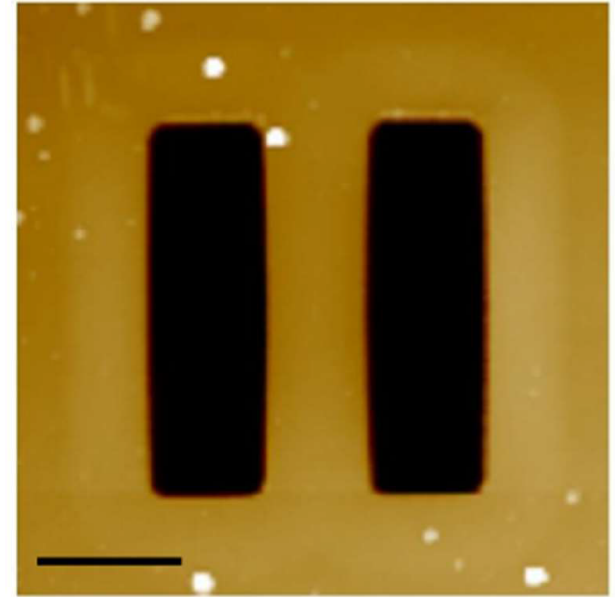

(c)

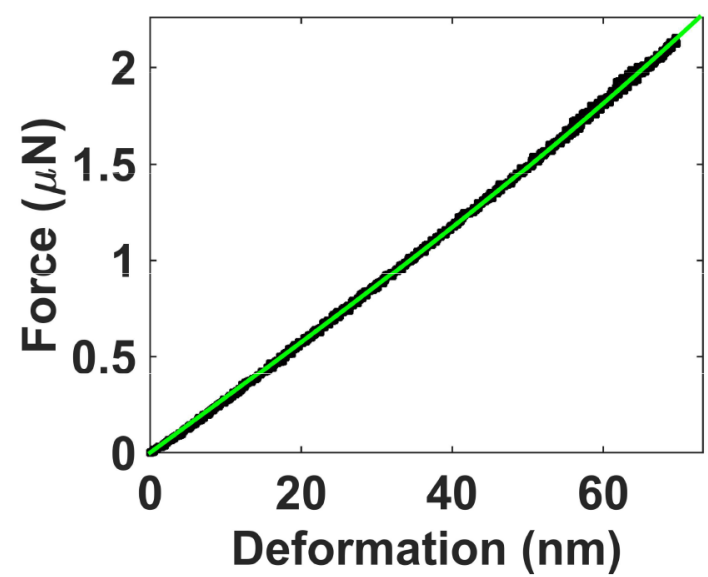

(d)

Fig. 2. (a) Schematic of the AFM-based central indentation method. (b) Equivalent spring-mass model of the system - $K_{c}$, $K_{i}$, and $\mathrm{K}_{m}$ are the stiffness of the AFM cantilever, tip-sample interaction, and membrane, respectively. $\mathrm{K}_{i}$ is a variable stiffness. $\mathrm{F}$ is the applied force, $\mathrm{M}_{c}$ and $\mathrm{M}_{m}$ are the effective mass of the cantilever and the membrane, respectively. (c) AFM topography micrograph of $5 \mu \mathrm{m}$ long and $1.3 \mu \mathrm{m}$ wide membrane fabricated from a $51 \mathrm{~nm}$ thick silicon nitride film. Scale bar is $2 \mu \mathrm{m}$. (d) Fitted Force vs. Deformation curve obtained on the same membrane after central indentation experiment.

on-substrate thin films. Stress in these on-substrate films was measured using the beam deflection technique [16] that considers the change in curvature of $\mathrm{Si} / \mathrm{SiO}_{2}$ substrate due to deposition of $\mathrm{SiN}_{\mathrm{x}}$ film. Subsequently, we use Stoney's equation [24] to determine the net stress in the $\mathrm{SiN}_{\mathrm{x}}$ film. Due to a high deposition temperature of $750^{\circ} \mathrm{C}$, thermal stress is developed in the film when it cools down to room temperature. So, the net stress in the film is the summation of thermal and intrinsic stress. We calculate the thermal stress [16] in the film as given below.

$$
\sigma_{t h}=\frac{E_{f}\left(\alpha_{s}-\alpha_{f}\right) \Delta T}{\left(1-\vartheta_{f}\right)}
$$

where $\sigma_{t h}$ is the thermal stress in the film; $E_{f}, \alpha_{f}$ and $\vartheta_{f}$ are the elastic modulus, coefficient of thermal expansion, and Poisson's ratio of the $\mathrm{SiN}_{\mathrm{x}}$ film; $\alpha_{s}$ is the coefficient of thermal expansion of the $\mathrm{Si} / \mathrm{SiO}_{2}$ substrate. $\Delta T$ is the difference between the deposition temperature and room temperature. For calculations, we use the measured value of $E_{f}$ using nanoindentation method, and average values of $\alpha_{f}=3.2 \times 10^{-6} /{ }^{\circ} \mathrm{C}\left(\right.$ for $\mathrm{SiN}_{\mathrm{x}}$ ) and $\alpha_{s}=0.65 \times 10^{-6} /{ }^{\circ} \mathrm{C}$ (for $\left.\mathrm{SiO}_{2}\right)[25,26]$. It is clear from equation (3) that the thermal stress will be more significant in a film with higher elastic modulus than in a film with lower elastic modulus. We calculate the intrinsic stress in a film by subtracting the thermal stress from the net stress.

\section{Results and discussion}

\subsection{Stress in suspended $\mathrm{SiN}_{x}$ membranes}

The elastic modulus of films of thickness 47 and $51 \mathrm{~nm}$ was very low, i.e., about $10 \%$ of the bulk modulus value of $270 \mathrm{GPa}$ [27], while that of $88 \mathrm{~nm}$ thick film was about $70 \%$ of the bulk value. However, the net stress was almost equal to $1.5 \mathrm{GPa}$ in all three films (Tab. 1). The LPCVD processparameters were the same for all three films except for the 
Table 1. Elastic modulus and stress in the on-substrate $\mathrm{SiN}_{\mathrm{x}}$ thin films.

\begin{tabular}{|c|c|c|c|c|}
\hline $\begin{array}{l}\text { Thickness of } \\
\text { deposited } \mathrm{SiN}_{\mathrm{x}} \\
\text { film } \\
t_{f}(\mathrm{~nm})\end{array}$ & $\begin{array}{l}\text { Elastic modulus of } \\
\text { on-substrate films } \\
\text { (Nanoindentation } \\
\text { method) } \\
E_{f}(\mathrm{GPa})\end{array}$ & $\begin{array}{l}\text { Net stress in } \\
\text { on-substrate films } \\
\text { (Beam deflection } \\
\text { method) } \\
\sigma_{f}(\mathrm{GPa})\end{array}$ & $\begin{array}{l}\text { Thermal stress in } \\
\text { on-substrate films } \\
\text { (Calculated using } \\
\text { Eq. }(3)) \\
\sigma_{t h}(\mathrm{GPa})\end{array}$ & $\begin{array}{l}\text { Intrinsic stress in } \\
\text { on-substrate films } \\
\text { (net stress - thermal } \\
\text { stress) } \\
\sigma_{i}(\mathrm{GPa})\end{array}$ \\
\hline 47 & $20.54 \pm 1.985$ & $1.432 \pm 0.114$ & 0.054 & 1.378 \\
\hline 51 & $28.52 \pm 0.528$ & $1.530 \pm 0.190$ & 0.075 & 1.455 \\
\hline 88 & $184.5 \pm 13.27$ & $1.627 \pm 0.259$ & 0.487 & 1.140 \\
\hline
\end{tabular}

Table 2. Elastic modulus and stress of the $5 \mu \mathrm{m}$ long $\mathrm{SiN}_{\mathrm{x}}$ suspended membranes measured using AFM.

\begin{tabular}{|c|c|c|c|c|}
\hline $\begin{array}{l}\text { Thickness of deposited } \\
\text { on-substrate } \mathrm{SiN}_{\mathrm{x}} \\
\text { film } \\
t_{f}(\mathrm{~nm})\end{array}$ & $\begin{array}{l}\text { Thickness of } \mathrm{SiN}_{\mathrm{x}} \\
\text { suspended } \\
\text { membrane } \\
t_{m}(\mathrm{~nm})\end{array}$ & $\begin{array}{l}\text { Elastic modulus } \\
\text { of suspended } \\
\text { membrane } \\
E_{m}(\mathrm{GPa}) \\
\end{array}$ & $\begin{array}{l}\text { Stress in } \\
\text { suspended } \\
\text { membrane } \\
\sigma_{m}(\mathrm{MPa}) \\
\end{array}$ & $\begin{array}{l}\text { Spring constant } \\
\text { of suspended } \\
\text { membrane } \\
k_{m}(\mathrm{~N} / \mathrm{m}) \\
\end{array}$ \\
\hline 47 & 8 & $104.1 \pm 1.2$ & $241.7 \pm 1.5$ & 0.233 \\
\hline 51 & 30 & $222.6 \pm 9.1$ & $861.8 \pm 2.7$ & 1.845 \\
\hline 88 & 45 & $204.1 \pm 16.9$ & $552.7 \pm 4.5$ & 3.260 \\
\hline
\end{tabular}

gas flow ratio $\left(\mathrm{NH}_{3}: \mathrm{H}_{2} \mathrm{Cl}_{2} \mathrm{Si}\right)$. For the $51 \mathrm{~nm}$ thick film, the gas flow ratio was 10:100, while for the other two films, the ratio was 10:70. Increasing the gas flow ratio from 1:2 to 1:20 for nitrogen-rich LPCVD SiN $_{\mathrm{x}}$ films has a negligible effect on the residual stress [28,29]. This explains why the net stress in all three films was almost equal. The contribution of thermal stress to the net stress was negligible for the 47 and $51 \mathrm{~nm}$ thick films due to their low elastic moduli. For the $88 \mathrm{~nm}$ thick film, thermal stress was about $30 \%$ of the total stress due to its higher elastic modulus. The thickness of suspended membranes (Tab. 2) was significantly lower than the corresponding on-substrate film's thickness (Tab. 1) as $\operatorname{SiN}_{\mathrm{x}}$ also gets etched during the wet etching of sacrificial $\mathrm{SiO}_{2}$ with BOE. We express this reduction in thickness after the fabrication of the suspended membrane by a ratio $\Delta t$ given by equation (4). The values of $\Delta t$ are $0.83,0.42$, and 0.49 for the on-substrate films of thickness 47,51 , and $88 \mathrm{~nm}$, respectively. A high value of $\Delta t$ indicates that a significant fraction of the highly-stressed region at the bottom of onsubstrate $\mathrm{SiN}_{\mathrm{x}}$ film is etched during the fabrication of suspended membrane.

$$
\Delta t=\frac{t_{f}-t_{m}}{t_{f}} .
$$

Tables 1 and 2 show that stress values in the suspended membranes were significantly lower than those in the corresponding on-substrate films. This reduction in stress after the fabrication of suspended membrane was due to thermal and intrinsic stress relaxation. The thermal stress relaxes because the suspended membrane was no longer attached to the substrate except its clamped ends. The relaxation of thermal stress was negligible in suspended membranes of thickness $8 \mathrm{~nm}$ and $30 \mathrm{~nm}$, while a $487 \mathrm{MPa}$ of thermal stress was relaxed in the $45 \mathrm{~nm}$ thick suspended membrane. The relaxation of intrinsic stress depends on the value of $\Delta t$ for the particular suspended membrane. The value of $\Delta t$ for the suspended membrane of thickness $8 \mathrm{~nm}$ was 0.81 , the highest amongst all three suspended membranes. It implies that the fabrication of the suspended membrane etches most of the highly stressed regions near the substrate of the $47 \mathrm{~nm}$ thick $\mathrm{SiN}_{\mathrm{x}}$ film. Hence, there was a drastic reduction in stress from $1.432 \mathrm{GPa}$ (for $47 \mathrm{~nm}$ thick on- substrate film) to $242 \mathrm{MPa}$ (for $8 \mathrm{~nm}$ thick suspended membrane). Simple calculations reveal that intrinsic stress relaxation in the suspended membranes of thickness $30 \mathrm{~nm}$ ( $51 \mathrm{~nm}$ thick on-substrate film) and $45 \mathrm{~nm}$ (88 nm thick on-substrate film) was 593 and $587 \mathrm{MPa}$, respectively. The relatively close values of 0.42 and 0.49 of $\Delta t$ for these two suspended membranes also indicate nearly equal intrinsic stress relaxation in them.

\subsection{Elastic modulus of suspended $\mathrm{SiN}_{\mathrm{x}}$ membranes}

Tables 1 and 2 also show that the elastic moduli of the suspended membranes were significantly higher than those of the corresponding on-substrate films. The $30 \mathrm{~nm}$ thick suspended membrane had the highest elastic modulus of $222 \mathrm{GPa}$. We expect that the calculated elastic moduli of the suspended membranes are slightly greater than the actual value. It is mainly due to two reasons: surface effects and offset of the tip from the center of the suspended membrane during indentation. Surface effects include the repulsive interaction forces between the tip and surface of the specimen that leads to a higher value of elastic modulus than the one obtained from nanoindentation techniques [30]. Thermal drift in the piezo and inclined motion of the tip creates an offset of few tens of nanometers from the 
Table 3. Limit thickness $\left(t_{0}\right)$ and strain $\left(\varepsilon_{0}\right)$ values for the suspended membranes.

\begin{tabular}{|c|c|c|c|c|}
\hline $\begin{array}{l}\text { Thickness of the } \\
\text { suspended membrane } \\
t_{m}(\mathrm{~nm})\end{array}$ & $\begin{array}{l}\text { Strain in the } \\
\text { suspended } \\
\text { membrane } \\
\varepsilon_{0}\left(\times 10^{-3}\right)\end{array}$ & $\begin{array}{l}\text { Limit thickness } \\
t_{0}(\mathrm{~nm})\end{array}$ & $\begin{array}{l}\text { Normalized thickness } \\
\text { of the suspended } \\
\text { membrane } \\
N_{t}=\frac{t_{m}}{t_{0}}\end{array}$ & $\begin{array}{l}\text { Normalized elastic modulus } \\
\text { of the suspended } \\
\text { membrane } \\
N_{E}=\frac{E_{m}}{E_{b u l k}}\end{array}$ \\
\hline 8 & 2.7 & 347.47 & 0.023 & 0.328 \\
\hline 30 & 4.6 & 124.97 & 0.240 & 0.700 \\
\hline 45 & 3.2 & 255.43 & 0.176 & 0.642 \\
\hline
\end{tabular}

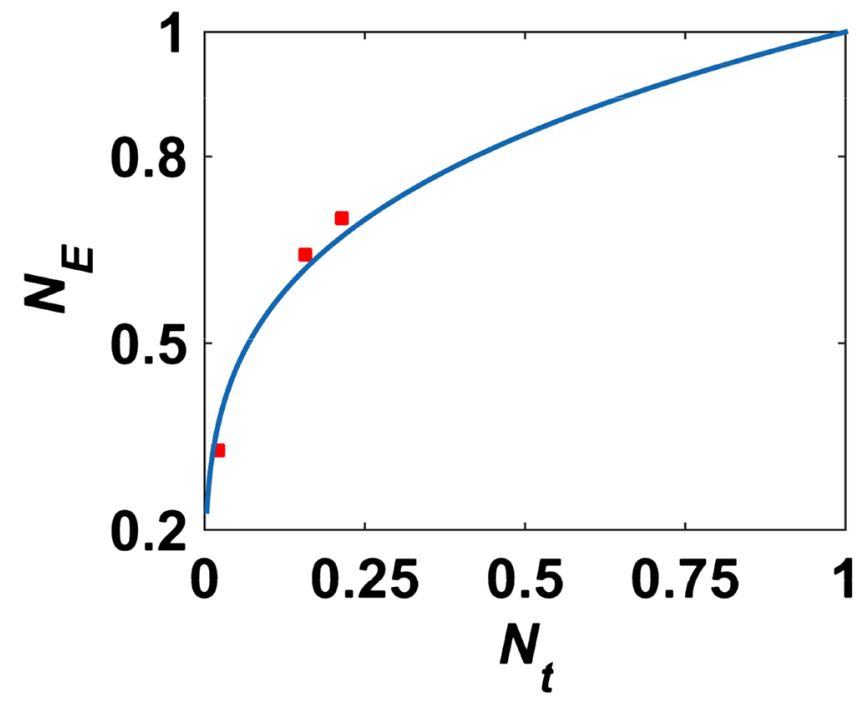

Fig. 3. Normalized elastic modulus vs. Normalized thickness of the suspended membrane.

center of the suspended membrane. The tip's motion is not in a direction perpendicular to the suspended membrane's surface due to the tip-sample repulsive forces. All these effects lead to a higher value of calculated elastic modulus. We further investigate the elastic modulus of suspended membranes using an approach similar to the one followed by Fedorchenko et al. [31]. A suspended membrane has both the top and bottom surfaces free, and therefore for surface energy calculations, we consider it as a thin film with both surfaces free. Equation (5) gives the limit thickness $t_{0}$ below which such a film cannot be considered as a bulk material [31].

$$
t_{0}=\frac{\left(1-\vartheta_{f}\right)}{E_{b u l k} \varepsilon_{0}^{2}}
$$

where $\vartheta_{f}$ is the Poisson's ratio of the film $=0.3$ (assumed), $E_{b u l k}$ is the bulk elastic modulus of $\mathrm{SiN}_{\mathrm{x}}$ and $\varepsilon_{0}$ is the strain in the film.

Table 3 lists the calculated values of $t_{0}$ and $\varepsilon_{0}$ for the suspended membranes. The strain in all the suspended membranes was not equal. Hence, we define a normalized thickness of a suspended membrane $\left(N_{t}\right)$ as the ratio of the thickness of suspended membrane and the limit thickness. Similarly, the normalized elastic modulus of a suspended membrane $\left(N_{E}\right)$ is also defined. The $30 \mathrm{~nm}$ thick suspended membrane has the highest normalized thickness; hence it has the highest elastic modulus among all the three suspended membranes. The variation in $N_{E}$ vs. $N_{t}$ can be expressed using equation (6).

$$
N_{E}=\left(N_{t}\right)^{p} .
$$

Curve fitting in (Fig. 3) yields a value of $0.27 \pm 0.06$ for $\mathrm{p}$. For any value of strain and thickness of the suspended membrane, equation (6) can be used to determine the elastic modulus of the suspended membrane.

\subsection{Collapse of suspended membranes}

The suspended membranes of thickness $30 \mathrm{~nm}$ collapsed to the bottom substrate under large applied central loads. The collapse of the suspended membranes is most likely due to pull-in through capillary adhesion force. Due to a tiny gap, a liquid bridge (capillary) is formed between the surface of a suspended membrane and the bottom substrate when they are very close to each other [32,33]. The pull-in of the suspended membranes is illustrated in the force-separation curves in Figures 4a-4c. Table S2 of Supplementary Information lists the several experimental parameters of the pull-in measurements. A sudden drop in force in the force-separation curve corresponds to the point of collapse of the suspended membrane. Such an abrupt drop in force was due to the cantilever spring relaxation as the adhesive force gradients pull the membrane to the substrate. During the pull-in of the suspended membrane, the $\mathrm{Z}$ position of piezo did not change as the pull-in process took less than $1 \mathrm{~ms}$ time. The pull-in process time $\left(t_{c}\right)$ is three orders of magnitude greater than the inverse of the fundamental resonance frequency of the suspended membranes $\left(1 / f_{0}\right)$. Hence, the dynamics of pull-in of the suspended membranes is governed by viscous damping.

The net relaxation of the AFM cantilever in the region $\mathrm{R}$ (Figs. 4d-4f) is equal to the gap between the suspended membrane and substrate at the start of the pull-in process. For the suspended membranes of length 20 and $40 \mu \mathrm{m}$, the AFM cantilever did not relax entirely after the pull-in process was over (stage (iv) in Fig. 5a). While for the $60 \mu \mathrm{m}$ long suspended membrane, the cantilever relaxed completely before the pull-in process was completed and then lost contact with the suspended membrane, thereby leading to a tiny gap between the tip and collapsed membrane. As a result, capillary condensation occurred in this tiny gap (stage (iv) in Fig. 5b), leading to snap-in of the AFM cantilever on the collapsed membrane (stage (v) in Fig. 5b). For all three suspended membranes, the piezo moves down in the $\mathrm{Z}$ direction and the tip presses down on the hard $\mathrm{SiN}_{\mathrm{x}}$ on Si substrate after the pull-in process is 


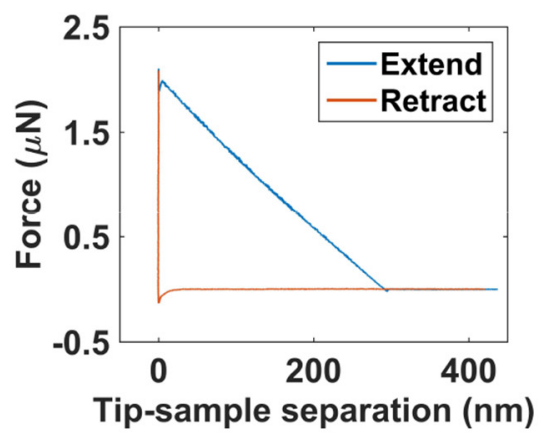

(a)

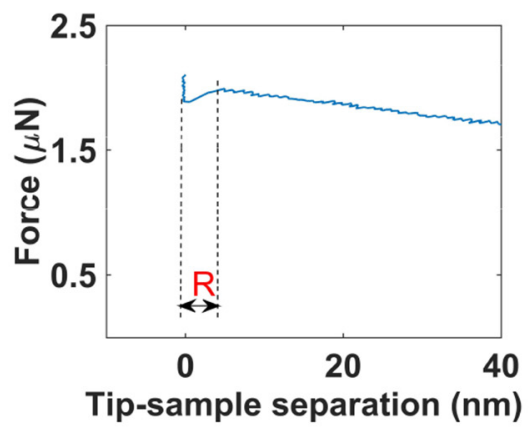

(d)

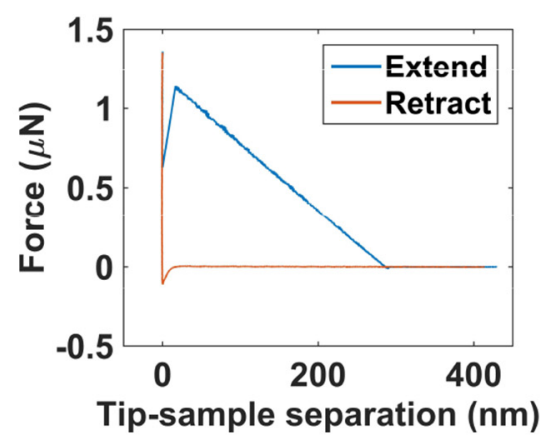

(b)

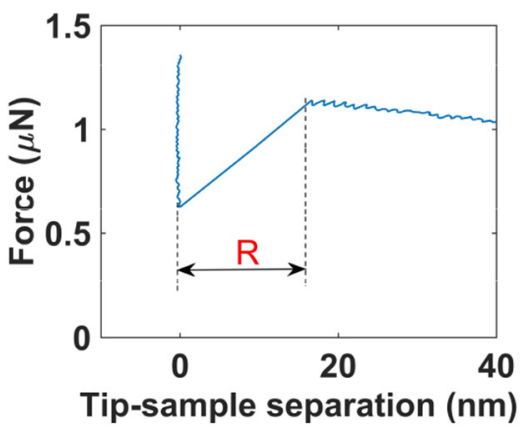

(e)

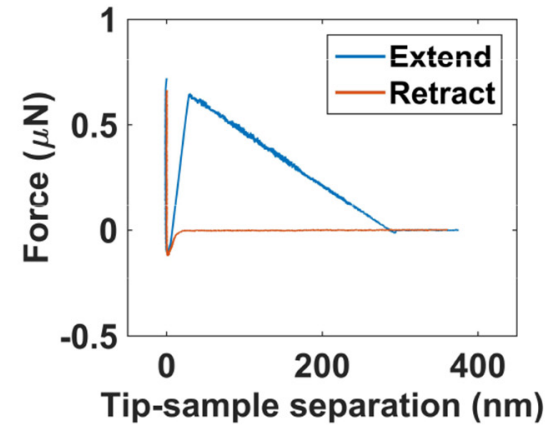

(c)

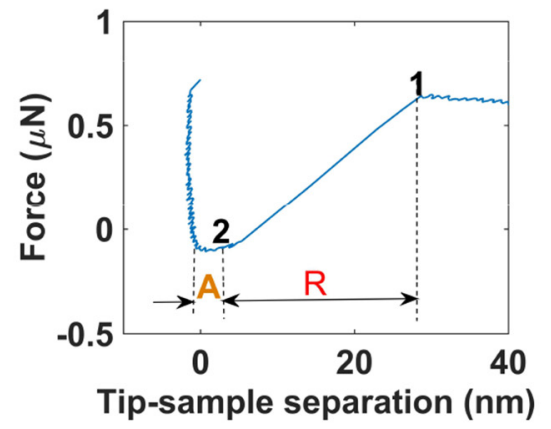

(f)

Fig. 4. Force vs. tip-sample separation curves indicating the collapse of $30 \mathrm{~nm}$ thick suspended membrane. (a) For $20 \mu \mathrm{m}$ long suspended membrane. (b) For $40 \mu \mathrm{m}$ long suspended membrane. (c) For $60 \mu \mathrm{m}$ long suspended membrane. (d-f) Zoomed region of the Force vs. tip-sample separation extend curve corresponding to the pull-in of suspended membranes of length 20 , 40 , and $60 \mu \mathrm{m}$, respectively. The slope of the curve in region $\mathrm{R}$ is equal to the spring constant of the cantilever.

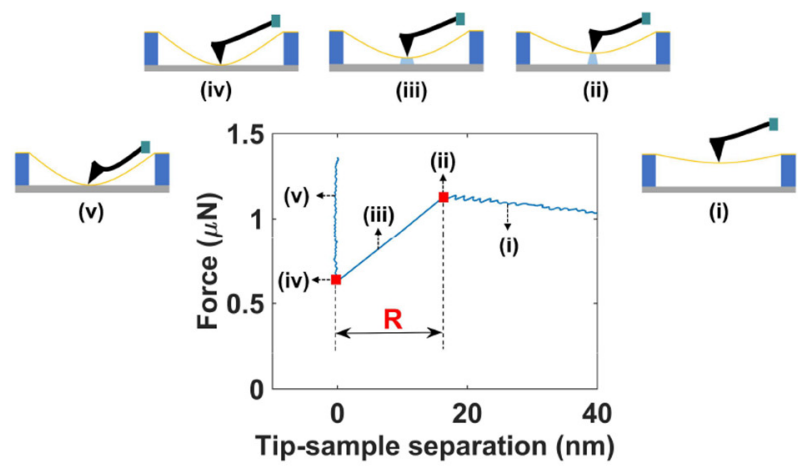

(a)
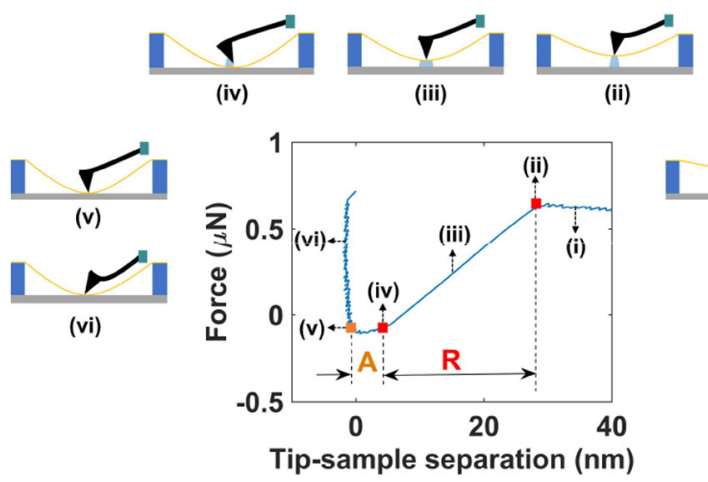

(b)

Fig. 5. (a) Pull-in process illustrated for suspended membranes of length 20 and $40 \mu \mathrm{m}$ based on the force-separation curve for $40 \mu \mathrm{m}$ long suspended membrane. (b) Pull-in process illustrated for 60 long suspended membrane based on its force-separation curve.

over. Only the AFM cantilever deflects during this process, so we observe an infinite slope in the force-separation curves. The cantilever continues to deflect until the specified force setpoint (stage (v) and (vi) in Figs. 5a and $5 \mathrm{~b}$, respectively). After this point, the piezo retracts, and the cantilever pulls off from the $\mathrm{SiN}_{\mathrm{x}}$ surface by overcoming the adhesion force.
A suspended membrane collapses when the sum of capillary force gradient and stiffness of the suspended membrane becomes zero, as given in equation (8). We calculate the stiffness of suspended membrane as negative of the slope of Force vs. tip-sample separation curve in the region just before the collapse of the suspended membrane. We fit the obtained Capillary force gradient vs. gap data 


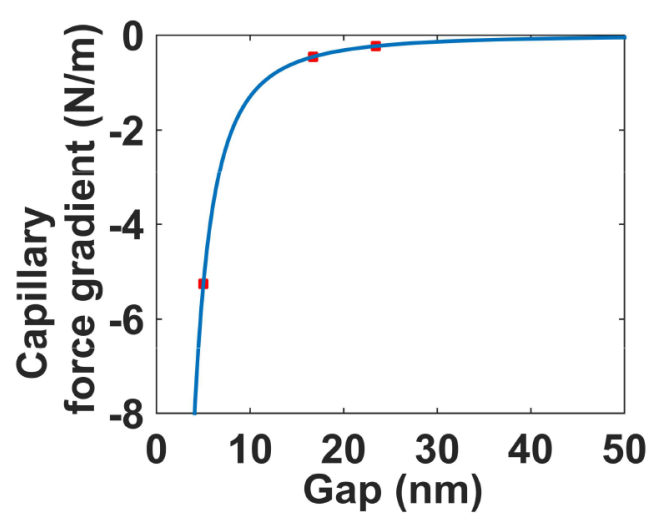

(a)

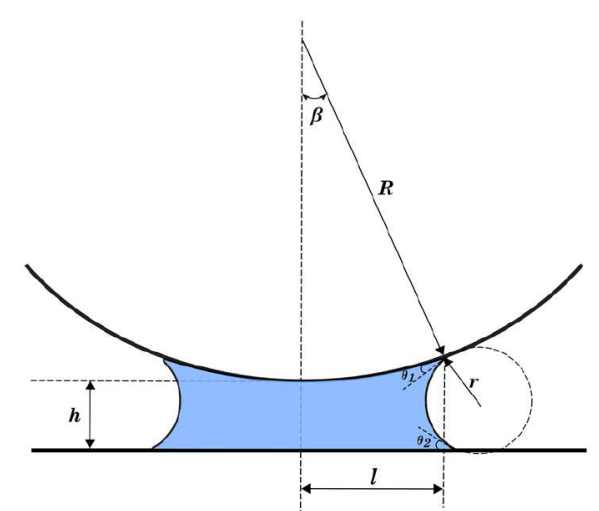

(b)

Fig. 6. (a) Capillary force gradient vs. Gap between the suspended membrane and the bottom substrate. (b) Schematic of a model for the capillary force between a sphere and a flat plate.

using a power relation (Eq. (9)), as shown in Figure 6a.

$$
\begin{gathered}
\frac{\partial F}{\partial h}+K_{m}=0 \\
\frac{\partial F}{\partial h}=-138.6 h^{-2.03 \pm 0.001}
\end{gathered}
$$

where, $\frac{\partial F}{\partial h}$ is the capillary adhesion force-gradient, $K_{m}$ is the stiffness of the suspended membrane, and $h$ is the gap between the $\mathrm{SiN}_{\mathrm{x}}$ suspended membrane and the bottom $\mathrm{Si}$ substrate.

Integration of equation (9) shows that the capillary force varies as $\frac{1}{h^{1.03}}$ with the distance between the suspended membrane and bottom substrate. Consider a section parallel to a suspended membrane's width and passing through its center. For this cross-section, the suspended membrane's central portion has a finite radius of curvature because the suspended membrane's width $(\sim 1 \mu \mathrm{m})$ is comparable to its deflection $(\sim 300 \mathrm{~nm})$. Therefore, an expression for the capillary force between the suspended membrane and bottom substrate can be determined analytically using a sphere and flat plate model shown in Figure 6b [34]. Comparing the analytical expression (for small $\beta$ ) and experimental fit gives $R \sin \beta=12.293$, where $\mathrm{R}$ is in nm. Thus, a known value of either the radius of curvature of suspended membrane $(R)$ or the filling angle $(\beta)$ would be sufficient to determine the variation of capillary force with the gap between the suspended membrane and bottom substrate.

\section{Conclusions}

In summary, we fabricated silicon nitride suspended membranes of thickness 8,30 , and $45 \mathrm{~nm}$ from LPCVD high-stress $\operatorname{SiN}_{\mathrm{x}}$ films of thickness 47,51 , and $88 \mathrm{~nm}$, respectively. Using the AFM-based indentation method, we studied the variation in elastic modulus and the stress for three different thicknesses of $5 \mu \mathrm{m}$ length suspended membrane. Thermal stress in the on-substrate films gets relaxed after the fabrication of the suspended membrane, while intrinsic stress relaxation depends on the fractional reduction in thickness $(\Delta t)$ after the fabrication of the suspended membrane. Higher the value of $\Delta t$, the higher the relaxation of intrinsic stress after fabrication of the suspended membrane. We use a model that considers the effect of the strain and ultralow thickness on the elastic modulus of the suspended membrane. Understanding this variation of elastic modulus and stress in the suspended membranes is essential for developing more efficient NEMS resonators. Finally, the collapse of $30 \mathrm{~nm}$ thick suspended membranes under large deformation is explained based on the capillary condensation and force-separation curves. Furthermore, we found that the capillary force-gradient varies inversely as the square of the gap between the suspended membrane and bottom substrate. This result can be constructive in the design and fabrication of suspended membrane-based NEMS devices to prevent failure due to condensation-induced capillary adhesive forces.

\section{Supplementary Material}

The Supplementary Material is available at https://www. epjap.org/10.1051/epjap/2021210068/olm.

We acknowledge funding support from DST SERB through grant number EMR/2016/006479 and from LAM Research through the 2018 Unlock ideas campaign. We also acknowledge funding support from MHRD, MeitY, and DST Nano Mission through NNetRA. We thank Prosenjit Sen for helpful technical discussions.

\section{Author contribution statement}

All authors discussed the results and contributed to the final manuscript. A.N. conceived the idea. A.A. fabricated the devices. S.J. performed the measurements and analyzed the data. S.J. and A.N. wrote the manuscript. 


\section{Appendix A \\ A.1 Fabrication of $\operatorname{SiN}_{x}$ suspended membranes}

The steps involved in the fabrication process were: (i) RCA cleaning of the $\mathrm{Si} / \mathrm{SiO}_{2}$ substrate. (ii) Deposition of LPCVD grown high-stress silicon nitride thin film on the substrate. Table S1 of Supplementary Information lists the process parameters. (iii) Spin-coating of PMMA A4 photoresist, followed by patterning suspended membrane features for different dimensions using Electron beam lithography and then developing photoresist using MIBK: IPA (1:3) developer. (iv) Reactive-ion-etching of silicon nitride from the windows opened in the lithography step using $\mathrm{SF}_{6}$ gas. (v) Removal of PMMA using oxygen plasma. (vi) Wet etching of sacrificial silicon dioxide using 13:2 buffered oxide etch (BOE/BHF), followed by Critical Point Drying (CPD) to release the suspended membranes.

\section{A.2 AFM characterization of $\mathrm{SiN}_{\mathrm{x}}$ suspended membranes}

The topography of the suspended membranes was acquired in AFM by scanning with a constant force of $10-40 \mathrm{nN}$ in Peak Force Quantitative Nanomechanical Mapping (PFQNM) [35] mode in Bruker Dimension Icon AFM. We determine the suspended membrane's length and width from the topography micrograph, while its thickness from the acquired force-distance curves. We apply a large force of about $10 \mu \mathrm{N}$ to the suspended membrane during the AFM scan. This force was sufficient to break the suspended membrane. We then measure the step height in the region where the fractured suspended membrane is in contact with the bottom Si substrate. The thickness of a suspended membrane was uniform in a large area of fractured portions of the suspended membrane in contact with the bottom substrate.

\section{A.3 Measurement of thickness of $\operatorname{SiN}_{x}$ thin films}

The thickness of $\mathrm{SiN}_{\mathrm{x}}$ films and $\mathrm{SiO}_{2}$ layer was measured using a Variable Angle Spectroscopic Ellipsometer (VASE) [36]. J.A. Woollam Co. M2000U. We perform the measurements at an incident angle of $65^{\circ}, 70^{\circ}$, and $75^{\circ}$. The thickness of the $\mathrm{SiO}_{2}$ layer is $300 \mathrm{~nm}$.

\section{A.4 Measurement of elastic modulus and stress in on-substrate $\operatorname{SiN}_{x}$ thin films}

The elastic moduli of the silicon nitride thin films were measured using the nanoindentation technique. Hysitron TI 950 Triboindenter with a Hysitron spherical diamond tip (radius $1 \mu \mathrm{m}$ ) is used to indent the sample in load control mode. We acquire loading and unloading Force vs.
Displacement curves in a load control of 100, 180, and $500 \mu \mathrm{N}$ for the films of thickness 47,51 , and $88 \mathrm{~nm}$, respectively. We obtain the elastic modulus by fitting the unloading curve using the Oliver-Pharr model $[37,38]$. We use the beam deflection technique in a laser-based kSA Multi-beam Optical Sensor (MOS) system to measure the change in substrate curvature and then relate it to stress in the on-substrate film using Stoney's equation.

\section{References}

1. A.K. Naik, M.S. Hanay, W.K. Hiebert, X.L. Feng, M.L. Roukes, Towards single-molecule nanomechanical mass spectrometry, Nature Nanotech. 4, 445 (2009)

2. M.S. Hanay et al., Single-protein nanomechanical mass spectrometry in real time, Nature Nanotech. 7, 602 (2012)

3. J. Chaste, A. Eichler, J. Moser, G. Ceballos, R. Rurali, A. Bachtold, A nanomechanical mass sensor with yoctogram resolution, Nature Nanotech. 7, 301 (2012)

4. W. Hiebert, Mass sensing: devices reach single-proton limit, Nature Nanotech. 7, 278 (2012)

5. S.S. Verbridge, H.G. Craighead, J.M. Parpia, A megahertz nanomechanical resonator with room temperature quality factor over a million, Appl. Phys. Lett. 92, 4 (2008)

6. S.S. Verbridge, D.F. Shapiro, H.G. Craighead, J.M. Parpia, Macroscopic tuning of nanomechanics: substrate bending for Reversible control of frequency and quality factor of nanostring resonators, Nano Lett. 7, 1728 (2007)

7. T. Larsen et al., Ultrasensitive string-based temperature sensors, Appl. Phys. Lett. 98, 121901-1 (2011)

8. S. Schmid, M. Kurek, J.Q. Adolphsen, A. Boisen, Real-time single airborne nanoparticle detection with nanomechanical resonant filter-fiber, Sci. Rep. 3, 1288-1 (2013)

9. I.W. Frank, D.M. Tanenbaum, A.M. van der Zande, P.L. McEuen, Mechanical properties of suspended graphene sheets, J. Vac. Sci. Technol., B 25, 2558 (2007)

10. S.S. Verbridge, J.M. Parpia, R.B. Reichenbach, L.M. Bellan, H.G. Craighead, High quality factor resonance at room temperature with nanostrings under high tensile stress, J. Appl. Phys. 99, 124304-1 (2006)

11. P.S. Alexopoulos, T. O'Sullivan, Mechanical properties of thin films, Annu. Rev. Mater. Sci. 20, 391 (1990)

12. L. Šimůrka, S. Erkan, T. Turutoglu, Characterization of silicon nitride thin films on glass, Defect Diffus. Forum 368, 86 (2016)

13. H. Huang et al., Effect of deposition conditions on mechanical properties of low-temperature PECVD silicon nitride films, Mater. Sci. Eng., A 435-436, 453 (2006)

14. B.C. Joshi, G. Eranna, D.P. Runthala, B.B. Dixit, O.P. Wadhawan, P.D. Vyas, LPCVD and PECVD silicon nitride for microelectronics technology, Indian J. Eng. Mater. Sci. 7, $303(2000)$

15. J. Vlassak, Thin Film Mechanics, Cambridge: Harvard University, 298 (2004)

16. A.G. Evans, J.W. Hutchinson, The thermomechanical integrity of thin films and multilayers, Acta Metall. Mater. 43, 2507 (1995)

17. A. Castellanos-Gomez, V. Singh, H.S.J. Van Der Zant, G.A. Steele, Mechanics of freely-suspended ultrathin layered materials, Ann. Phys. 527, 27 (2015) 
18. C. Lee, X. Wei, J.W. Kysar, J. Hone, Measurement of the elastic properties and intrinsic strength of monolayer graphene, Science 321, 385 (2008)

19. B. Cappella, G. Dietler, Force-distance curves by atomic force microscopy, Surf. Sci. Rep. 34, 1 (1999)

20. H.J. Butt, B. Cappella, M. Kappl, Force measurements with the atomic force microscope: technique, interpretation and applications, Surf. Sci. Rep. 59, 1 (2005)

21. Y. Seo, W. Jhe, Atomic force microscopy and spectroscopy, Rep. Prog. Phys. 71, 016101-1 (2008)

22. S. Senturia, Microsystem Design. Boston, MA: Kluwer Academic, 2000

23. P.H. Wu, I.K. Lin, H.Y. Yan, K.S. Ou, K.S. Chen, X. Zhang, Mechanical property characterization of sputtered and plasma enhanced chemical deposition (PECVD) silicon nitride films after rapid thermal annealing, Sens. Actuators, A 168, 117 (2011)

24. G.C.A.M. Janssen, M.M. Abdalla, F. van Keulen, B.R. Pujada, B. van Venrooy, Celebrating the 100th anniversary of the Stoney equation for film stress: developments from polycrystalline steel strips to single crystal silicon wafers, Thin Solid Films 517, 1858 (2009)

25. AZO Materials, Properties: Silicon Nitride (Si3N4) Properties and Applications, AZO Materials, 2001. http://www. azom.com/properties.aspx?ArticleID $=53$

26. AZoM, Silica-Silicon Dioxide, AZO Materials, 2001. https:// www.azom.com/properties.aspx?ArticleID $=1114$

27. D.R. Askeland, F. Haddleton, P. Green, H. Robertson, The Science and Engineering of Materials (Springer, US, 1996)

28. J.G.E. Gardeniers, H.A.C. Tilmans, C.C.G. Visser, LPCVD silicon-rich silicon nitride films for applications in micromechanics, studied with statistical experimental design, J. Vac. Sci. Technol., A 14, 2879 (1996)

29. A.G. Noskov, E.B. Gorokhov, G.A. Sokolova, E.M. Trukhanov, S.I. Stenin, Correlation between stress and structure in chemically vapour deposited silicon nitride films, Thin Solid Films 162, 129 (1988)

30. H. Ni, X. Li, Young's modulus of $\mathrm{ZnO}$ nanobelts measured using atomic force microscopy and nanoindentation techniques, Nanotechnology 17, 3591 (2006)

31. A.I. Fedorchenko, A.B. Wang, H.H. Cheng, Thickness dependence of nanofilm elastic modulus, Appl. Phys. Lett. 94, 152111-1 (2009)

32. S. Cheng, M.O. Robbins, Capillary adhesion at the nanometer scale, Phys. Rev. E: Stat. Phys., Plasmas, Fluids, 89, 062402 (2014)

33. S. Cheng, M.O. Robbins, Nanocapillary adhesion between parallel plates, Langmuir 32, 7788 (2016)

34. H.-J. Butt, M. Kappl, Normal capillary forces, Adv. Colloid Interface Sci. 146, 48 (2009)

35. S.C. Minne, Y. Hu, S. Hu, B. Pittenger, C. Su, NanoScale quantitative mechanical property mapping using peak force tapping atomic force microscopy, Microsc. Microanal. 16, $464(2010)$

36. J.A. Woollam, P.G. Snyder, Fundamentals and applications of variable angle spectroscopic ellipsometry, Mater. Sci. Eng., B 5, 279 (1990)

37. J.A. Taylor, The mechanical properties and microstructure of plasma enhanced chemical vapor deposited silicon nitride thin films, J. Vac. Sci. Technol., A 9, 2464 (1991)

38. G.M. Pharr, W.C. Oliver, Measurement of thin film mechanical properties using nanoindentation, MRS Bull. 17, 28 (1992)

Cite this article as: Sanket S. Jugade, Anuj Aggarwal, Akshay K. Naik, Nanomechanical spectroscopy of ultrathin silicon nitride suspended membranes, Eur. Phys. J. Appl. Phys. 94, 20301 (2021) 